\title{
CIRUGÍA BARIÁTRICA EN MUJERES EN EDAD FÉRTIL. RECOMENDACIONES DE LOS COMITÉS DE TRABAJO DE “DIABETES $Y$ EMBARAZO" $Y$ "DIABETES Y OBESIDAD" DE LA SOCIEDAD ARGENTINA DE DIABETES
}

\author{
BARIATRIC SURGERY IN WOMEN IN REPRODUCTIVE AGE. \\ RECOMMENDATIONS FROM THE "DIABETES AND PREGNANCY" \\ AND "DIABETES AND OBESITY" WORKING COMMITTEES FROM \\ THE ARGENTINE SOCIETY OF DIABETES
}

Susana Gutt ${ }^{1}$, Gabriela Rovira ${ }^{2}$, Alicia Jawerbaum ${ }^{3}$, Frank Espinoza ${ }^{4}$, Juliana Mociulsky ${ }^{5}$, Stella Sucani ${ }^{6}$, Verónica Kojdamanian7, Marianela Aguirre Ackermann ${ }^{8}$, María Graciela Álvarez ${ }^{9}$, Alejandro Camaño ${ }^{10}$, Liliana Glatstein ${ }^{11}$, Paula Lifszyc ${ }^{12}$, Carolina Gómez Martin ${ }^{13}$, Patricio Mendes ${ }^{14}$, Estrella Menéndez ${ }^{15}$, María Yuma $^{16}$, Celina Bertona ${ }^{17}$, Lilia Cafaro ${ }^{18}$, Susana Fuentes ${ }^{19}$, Susana Salzberg ${ }^{20}$, Natalia Basualdo ${ }^{21}$, Sandra González ${ }^{22}$, Beatriz Villarroel Parra ${ }^{23}$, Carla Musso ${ }^{24}$

Coordinadores: Susana Gutt, Carla Musso, Gabriela Rovira

Revisores: Jorge Alvariñas ${ }^{25}$, Silvia Lapertosa ${ }^{26}$, León Litwak ${ }^{27}$, María Cristina Faingold ${ }^{28}$

\section{RESUMEN}

El incremento de la obesidad en el mundo, a edades más tempranas, ha tenido un impacto negativo especialmente en las mujeres en edad reproductiva. Se ven afectadas tanto la fertilidad como la tasa de embarazos de riesgo, de niños con bajo peso al nacer, pretérmino e índice de cesáreas.

La cirugía bariátrica como alternativa de tratamiento de la obesidad ha mejorado la fertilidad, pero el trastorno malabsortivo que provoca modifica la absorción de nutrientes en la madre y en el feto.

El diagnóstico de diabetes gestacional se dificulta dado que la prueba de tolerancia oral a la glucosa estaría contraindicada en estos casos.

El objetivo de estas recomendaciones es proveer herramientas para el control y seguimiento de mujeres en edad reproductiva sometidas a cirugía bariátrica.

Palabras clave: cirugía bariátrica, obesidad, embarazo, diabetes gestacional.

Revista de la Sociedad Argentina de Diabetes 2015; Vol. 49 (85-94)

\section{ABSTRACT}

Worldwide increased obesity prevalence at early ages particularly affects women of reproductive ages. Both fertility and risk pregnancy rates are affected, of low birth weight infants, prematures and cesarean section rates have been modified considerably.

Bariatric surgery is a validated tool to improve fertility but nutrient malabsorption as a consequence of surgery could affect mother and fetus nutrition and development.

Gestational diabetes diagnosis turns difficult in this group of obese patients after bariatric surgery since oral glucose tolerance testing would be contraindicated in these cases.

The aim of these recommendations was to offer tools for a good control and follow up of women at reproductive age.

Key words: bariatric surgery, obesity, pregnancy, gestational diabetes.

Revista de la Sociedad Argentina de Diabetes 2015; Vol. 49 (85-94) 


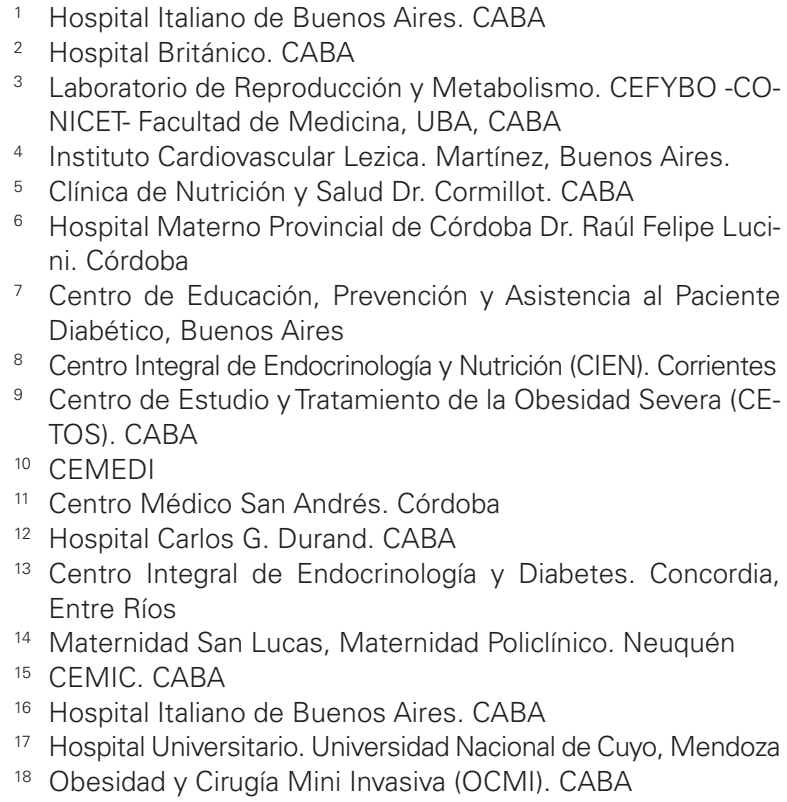

19 Hospital El Cruce. Buenos Aires

20 Instituto Centenario. CABA

21 Hospital Materno Infantil Ramón Sardá. CABA

22 Clínica IMA. Buenos Aires

23 Hospital de Agudos Dalmacio Vélez Sarsfield. Sanatorio Santa Isabel. CABA

24 Fundación Favaloro. CABA

${ }^{25}$ Consultor en Nutrición del Hospital Enrique Tornú. CABA

${ }^{26}$ Médica Endocrinóloga. Prof. Titular de Nutrición y Educación para la Salud, Facultad de Medicina, Universidad Nacional del Nordeste

27 Hospital Italiano de Buenos Aires. CABA

28 Servicio de Endocrinología de la Unidad Asistencial Dr. César Milstein. CABA

Contacto del autor: Susana Gutt

E-mail: Susana.gutt@gmail.com

Correspondencia: Av. Callao 1502 (C1024AAO), CABA,

Argentina

Tel.: (011) 15-6174-4824

Fecha de trabajo recibido: 17/08/15

Fecha de trabajo aceptado: 10/10/15

Conflictos de intereses: los autores declaran que no existen conflicto de intereses

\section{INTRODUCCIÓN}

El incremento en la prevalencia de obesidad en el mundo es alarmante y actualmente es el trastorno metabólico más común. La Organización Mundial de la Salud (OMS) considera a la obesidad como uno de los problemas más graves de salud mundial del siglo XXI'. La obesidad y el sobrepeso que se presentan previos y durante el embarazo constituyen un factor de riesgo importante para las complicaciones maternas y fetales durante la gestación, el parto y posteriores al parto ${ }^{2-4}$.

Cuando la obesidad ocurre en la mujer en edad reproductiva, tanto antes como durante el embarazo, confiere un mayor riesgo de complicaciones maternas como fetales ${ }^{2}$.

\section{Epidemiología}

En Argentina, según la Encuesta Nacional de Factores de Riesgo (ENFR) realizada en el año 2013, seis de cada personas 10 tienen sobrepeso u obesidad. La obesidad tiene una prevalencia del $20,8 \%$ en la población adulta. Discriminado en mujeres de edad fértil de 18 a 45 años, el sobrepeso, la obesidad y la obesidad mórbida alcanzan en conjunto un porcentaje del $42,2 \%{ }^{5}$.

Según la OMS, la prevalencia de obesidad en el embarazo tiene un rango que va del 1,8 al 25\% según la población estudiada². En la Encuesta Nacional de Nutrición y Salud realizada en Argentina, en 2005, la prevalencia de sobrepeso y obesidad en mujeres embarazadas fue del 19,7 y $24,4 \%$ respectivamente (Tabla 1).

\begin{tabular}{|c|l|l|l|l|}
\hline & Bajo peso & Normal & $\begin{array}{c}\text { Pre- } \\
\text { obesidad }\end{array}$ & Obesidad \\
\hline $\begin{array}{c}\text { Prevalencia } \\
(\%)\end{array}$ & 24,9 & 31,1 & 19,7 & 24,4 \\
\hline IC 95\% & $20,0-30,4$ & $25,9-36,8$ & $15,0-25,4$ & $19,6-29,9$ \\
\hline
\end{tabular}

Tabla 1: Estado nutricional antropométrico de embarazadas de 19 y más años de edad y entre las semanas 10 y 43 de gestación según la curva de Rosso-Mardones (datos de la Encuesta Nacional de Nutrición y Salud, 2005. http://datos.dinami.gov.ar/produccion/ nutricion/material/A1a.pdf).

La obesidad materna se asocia a complicaciones que impactan en el embarazo:

- Complicaciones tempranas: aborto espontáneo y aborto recurrente.

- Complicaciones tardías: limitaciones diagnósticas, síndrome metabólico, preeclampsia (OR 1.63.3), enfermedad hipertensiva del embarazo (OR 2.5 a 3.2) y diabetes gestacional. El riesgo de desarrollar diabetes gestacional aumenta 1,97 veces (95\% Cl 1.77-2.19), 3,01 veces (95\% Cl 2.34-3.87) y 5,55 veces $(95 \% \mathrm{Cl} 4.27$ 7.21) en sobrepeso, obesidad moderada y obesidad mórbida respectivamente ${ }^{4}$; también existe mayor riesgo de macrosomía (OR 1.7 a 1.9), parto pretérmino, muerte fetal intraútero y apnea obstructiva del sueño.

- Impacto en el parto: macrosomía y distocia de hombros, dificultad para realizar monitorización fetal, monitorización de la dinámica uterina y fases de dilatación, mayor índice de fracaso en la induc- 
ción del parto e incremento de partos por cesárea. Según el índice de masa corporal (IMC) la tasa de cesáreas fue de 20,7\% para mujeres con IMC de 29,9 ó menos, 33,8\% para mujeres con IMC de $30-34,9$ y $47,4 \%$ para mujeres con un IMC de 3539,96. También es dificultosa la anestesia obstétrica y existe mayor riesgo de tromboembolismo: el embarazo aumenta cuatro a cinco veces el riesgo de enfermedad tromboembólica?. El riesgo de trombosis venosa profunda y tromboembolismo pulmonar es mayor en el postparto. Durante las primeras seis semanas postparto el riesgo aumenta de 20 a 80 veces, y en la primera semana postparto esta cifra es cerca de 100 veces $^{8}$.

- Impacto en el puerperio: estancia hospitalaria prolongada, aumento del riesgo de infección puerperal y hemorragia posparto.

- Impacto en el feto y el neonato: anomalías congénitas como defectos del tubo neural (espina bífida OR 1.2-3.5), defectos cardíacos (OR 1.18-2), defectos en la pared abdominal, onfalocele, grandes para edad gestacional (mayor del percentilo 90), muerte intrauterina o neonatal ${ }^{4,9,5,10}$.

Conociendo que la cirugía bariátrica (CB) es un tratamiento para la obesidad mórbida, que su práctica está extendida y que más de la mitad de los casos en que se efectúa la paciente se encuentra en etapa reproductiva, es de interés abordar en esta guía las recomendaciones para que la mujer tenga los cuidados necesarios en forma preconcepcional, gestacional y en el postparto, en beneficio propio y de su descendencia.

\section{¿Qué es la cirugía bariátrica? Diferentes técnicas quirúrgicas}

La cirugía bariátrica (del griego baros=peso) es el tratamiento quirúrgico de la obesidad mórbida. Es una intervención sobre el tubo digestivo que logra el descenso del peso y su mantenimiento a largo plazo.

Los cuatro procedimientos que se utilizan en la actualidad inicialmente se clasificaron según sus mecanismos de acción restrictivos y/o malabsortivos. Dos técnicas son estrictamente restrictivas: la banda gástrica ajustable y la gastroplastía en manga o manga gástrica, una mixta (restrictiva-malabsortiva), el bypass gástrico en Y-Roux (BGYR) considerado la técnica "patrón de oro" del tratamiento de la obesidad y la restante es fundamentalmente malabsortiva, la derivación biliopancrática, con una variante: el switch duodenal ${ }^{11}$. El tipo de procedi- miento bariátrico es importante en relación a las posibles complicaciones gestacionales.

Es frecuente la presencia de vómitos y náuseas en mujeres que se sometieron a $\mathrm{CB}$, especialmente cuando la masticación no es prolongada o la ingesta alimentaria es rápida. Dado que las náuseas y vómitos en el primer trimestre de gestación son frecuentes relacionados con los altos niveles de $\beta \mathrm{HCG}$ o la disminución de los niveles de progesterona, debe considerarse la posibilidad de un embarazo.

\section{Se debe considerar que todas las técnicas de cirugía bariátrica no se hallan exentas de complicaciones en mujeres en edad re- productiva (A).}

\section{Programación del embarazo post-cirugía bariátrica}

El embarazo es un estado fisiológico normal en el que aumentan las demandas de nutrientes para sostener el crecimiento y desarrollo feto-placentario. En las mujeres con antecedente de una cirugía bariátrica (CB) y que programan un embarazo deben realizarse evaluaciones nutricionales en la etapa previa a la concepción y durante el embarazo hasta el parto.

\section{Ganancia de peso durante el embarazo}

La ganancia de peso durante el embarazo debe ser la misma que en la población general de acuerdo al IMC al inicio del embarazo.

\begin{tabular}{|l|l|}
\hline \multicolumn{1}{|c|}{ Estado nutricional previo } & \multicolumn{1}{|c|}{$\begin{array}{c}\text { Ganancia de peso } \\
\text { recomendada en el curso } \\
\text { del embarazo }\end{array}$} \\
\hline Bajo peso IMC $<19$ & 12,5 a $18 \mathrm{~kg}$ \\
\hline Normal IMC $19,9-24,9$ & 11 a $12,5 \mathrm{~kg}$ \\
\hline Sobrepeso IMC $24,9-29,9$ & $7 \mathrm{~kg}$ \\
\hline Obesidad IMC $>30$ & $7 \mathrm{~kg}$ \\
\hline
\end{tabular}

Tabla 2: Recomendaciones para la gestante con diabetes. Revista de la Sociedad Argentina de Diabetes. Vol. $43 \mathrm{~N}^{\circ}$ 2: 73-81, 2009.

La cirugía podría tener un efecto protector de ganancia excesiva de peso durante el embarazo ${ }^{14}$.

\section{Tiempos necesarios de espera antes de buscar embarazo}

Durante el primer año luego de la cirugía, 
la mujer se encuentra en fase catabólica: es el período donde se produce la mayor pérdida de peso alrededor del $30 \%$ del peso inicial luego del BGYR; hay alteraciones en la nutrición materna y esto podría acarrear riesgos en la salud fetal y neonatal ${ }^{13,14,15}$. Las pacientes se adecúan a los nuevos patrones alimentarios y hay cambios significativos de la imagen corporal; además puede resultar difícil diferenciar entre síntomas propios del embarazo y aquellos atribuibles a la cirugía tales como náuseas y vómitos o dolor abdominal16. Puede que aún no se haya logrado el descenso esperado de peso o la remisión completa de las comorbilidades de la obesidad, diabetes, hipertensión arterial y síndrome de apnea del sueño y este tiempo de espera ayuda a disminuir factores de riesgo presentes que puedan afectar el crecimiento y el desarrollo del feto. Si bien no existe una evidencia clara que demuestre mayor morbilidad fetal, cuando la gestación se produce durante el primer año posterior a la cirugía, algunos autores han encontrado mayor riesgo de parto prematuro y aborto espontáneo cuando los embarazos se producen dentro de los primeros 12 meses posteriores a una cirugía bariátrica ${ }^{17,18,19}$, mientras que otros autores comparando el resultado perinatal entre gestaciones tempranas (en los primeros 12 meses post $\mathrm{CB}$ ) versus tardías (> a 12 a 18 mes post $(\mathrm{B})$, no encontraron diferencias significativas al analizar las complicaciones obstétricas o neonatales en el crecimiento fetal temprano o el peso al nacer de acuerdo a la edad gestacional comparando entre estos dos grupos 20,21,22.

La mayoría de las recomendaciones coincide en evitar la gestación en los primeros 12 a 18 meses postoperatorios. Sin embargo algunos grupos no recomiendan esperar si el objetivo de la cirugía fue lograr un embarazo, sobre todo si utilizaron procedimientos restrictivos porque hay menos deficiencia de nutrientes que en el BGYR ${ }^{21,23}$.

Los embarazos no programados durante el primer año de la cirugía pueden explicarse por el aumento de la fertilidad posterior a la cirugía bariátrica o por la posible malabsorción de los anticonceptivos orales. Las pacientes que se embarazan dentro del primer año después de una CB necesitan una estricta vigilancia de su peso, estado alimenticio y crecimiento fetal' ${ }^{12}$.

Los embarazos, después de una CB, tienen menor incidencia de hipertensión gestacional, preclampsia y diabetes gestacional si se compara con el de mujeres obesas, pero los riesgos son mayores que en la población general no obesa $24,25,26,27$.

Al ser limitada y no concluyente la evidencia que apoya los diferentes enfoques, en algunos casos el consejo puede ser individualizado.

\section{Se aconseja evitar el embarazo en los pri- meros 12 a 18 meses posteriores a la ciru- gía bariátrica (C-IV) (opinión de expertos).}

\section{Recomendaciones nutricionales}

Cuando la mujer desea un embarazo es conveniente que sea programado para evitar la deficiencia de proteínas, vitaminas y minerales que puedan afectar la evolución de la gestación y al feto. Debe ser controlada por un equipo interdisciplinario, formado por diversos especialistas entre los que se encuentran: clínico especializado en obesidad o especialista en nutrición o endocrinólogo, obstetra, cirujano bariátrico, licenciados en nutrición, y si fuera necesario, psicólogo 28,29 .

En los casos en los cuales el embarazo no haya sido programado, el riesgo de déficit nutricional puede ser mayor, dado que de acuerdo a diversas publicaciones sólo el $50 \%$ de las pacientes mantiene adherencia a la suplementación vitamínica indicada en forma crónica.

Como parte del consejo previo a la concepción, se debe informar que la ingesta oral reducida y la malabsorción post CB pueden afectar los niveles de varios micronutrientes, que una correcta suplementación puede evitar complicaciones tanto en la madre como en el niño. Si el embarazo no fue programado tiene especial importancia la evaluación nutricional temprana, ya que son frecuentes los déficits de micronutrientes o de proteínas post-cirugía bariátrica especialmente luego de los procedimientos malabsortivos y mixtos ${ }^{20}$. Sin embargo, no se observó mayor incidencia de malformaciones congénitas asociadas a déficits nutricionales en embarazadas luego de la cirugía bariátrica ${ }^{20,30,31}$. Se detectó aumento del riesgo de recién nacidos pequeños para la edad gestacional (odds ratio 2, 1,5-2,5, $p<0,001$ ) $^{20,30,31}$ y de nacimientos pretérmino ${ }^{30,31}$; estos efectos persisten en embarazos subsiguientes luego de la cirugía ${ }^{20}$. El riesgo de recién nacidos pequeños para la edad gestacional y de parto pretérmino es menor en embarazadas con un IMC mayor a $35 \mathrm{~kg} / \mathrm{m}^{2}$ (Roos N. Perinatal outcomes after bariatric surgery: na- 
tionwide population based matched cohort study. $B M J, 2013)$. La opinión de expertos recomienda el control cercano y frecuente del crecimiento fetal durante el embarazo ${ }^{20}$. Para individualizar el tratamiento y ajustar las dosis según necesidad se debe monitorear hierro, transferrina, ferritina, folato, vitaminas A, B1, B6, B12, D, E, calcio zinc, selenio, magnesio y albúmina ${ }^{13,14}$ (Tabla 3). También deberían realizarse estudios de laboratorio que permitan conocer el estado de la función renal, hepática, el perfil lipídico e hidrocarbonado, electrolitos y hemograma.

La suplementación, que se inicia en el postoperatorio inmediato para la mayoría de los micronutrientes, dependerá de las condiciones fisiológicas y el procedimiento realizado, según modifiquen la ingesta de alimentos, su absorción o ambas. Su deficiencia debe ser detectada y corregida antes de la gestación.

La prevalencia de anemia es mayor en las embarazadas post-bariátricas en comparación con mujeres obesas no operadas, y en algunas series Ilega al 25-30\% ${ }^{38}$. Una vez descartadas las causas comunes de anemia -el déficit de hierro, de vitamina B12 ó ácido fólico- deben buscarse causas más raras como el déficit de cobre ${ }^{20}$. La deficiencia de hierro puede asociarse a partos pretérmino ${ }^{38}$ y para prevenir su déficit deberá indicarse hierro en forma ferrosa, en dosis mayor a las habituales durante el embarazo: 40-65 mg diarios; la suplementación se adecuará a los controles de laboratorio ${ }^{24,31}$. El déficit de vitamina B12 se asocia a hiperhomocisteinemia la cual se relaciona con la pérdida temprana del embarazo, también puede asociarse a trastornos neuroconductuales en la infancia y podría resultar en anemia en los lactantes; por ello en caso de déficit, debe suplementarse durante el embarazo diariamente con colabamina de fácil absorción, en dosis de 10 mcg por vía sublingual para mantener niveles adecuados de B12 y normalizar la homocisteína o también puede indicarse la suplementación por vía intramuscular en dosis mensuales de 1.000 mcg $^{30,31,32,33}$. El Consenso Argentino de Nutrición en Cirugía Bariátrica recomienda la suplementación de ácido fólico preconcepcional a lo largo de por lo menos un año previo a la concepción ${ }^{15}$.

Diversos estudios muestran que el déficit de vitamina $D(<20 \mathrm{ng} / \mathrm{dl})$ se asocia con parto pretérmino; su déficit puede asociarse a inflamación placentaria e incluso preeclampsia ${ }^{38}$. La deficien- cia de las vitaminas liposolubles A, D, E y K que puede observarse luego de cirugías malabsortivas son casos que deberán ser evaluados y corregidos previamente a la programación de un embarazo. El dosaje de otros micronutrientes como cobre, selenio y tiamina debe realizarse sólo si clínicamente se sospecha su déficit.

Se recomienda que todas las embarazadas post-cirugía bariátrica reciban uno o dos multivitamínicos diarios con 1.200-2.000mg de citrato de calcio, 400-800UI de vitamina D o dosis necesaria para alcanzar $>30 \mathrm{ng} / \mathrm{dl}, 400 \mathrm{mcg}$ de ácido fólico, 45-60 mg de hierro elemental evitando formulaciones de liberación prolongada como prevención y los casos de déficit pueden requerir la administración de hierro endovenoso ${ }^{34,38}$. La vitamina B12 debe administrarse diariamente en forma sublingual o mensualmente por vía intramuscular.

\begin{tabular}{|l|l|}
\hline \multicolumn{1}{|c|}{ Deficiencia } & \multicolumn{1}{c|}{ Efecto en el embarazo } \\
\hline Calcio & $\begin{array}{l}\text { Pérdida de masa ósea materna y } \\
\text { trastornos hipertensivos }\end{array}$ \\
\hline Vitamina D & Preeclampsia, parto prematuro \\
\hline Hierro & Anemia \\
\hline Vitamina B12 & $\begin{array}{l}\text { Anemia y } \uparrow \text { de homocisteina que } \uparrow \text { el } \\
\text { riesgo de aborto }\end{array}$ \\
\hline Ácido fólico & $\begin{array}{l}\text { Parto prematuro y defecto del tubo } \\
\text { neural }\end{array}$ \\
\hline Zinc & Parto prematuro \\
\hline Vitamina K & Trastornos hemorrágicos \\
\hline Magnesio & Preeclampsia y parto prematuro \\
\hline
\end{tabular}

Tabla 3: Recomendaciones nutricionales.

\section{Para disminuir el riesgo de complicaciones materno-fetales en mujeres que tuvieron una cirugía bariátrica se recomienda en el período previo a la concepción monito- rear albúmina, vitaminas y minerales para realizar una adecuada suplementación (ACOG) (C-IV).}

La nutrición materna durante el embarazo no sólo es relevante para el peso del RN y los resultados peri-parto, sino también para la salud del niño en su vida adulta, según la teoría de la programación intrauterina en la cual las influencias ambientales determinan la expresión de genes y circuitos metabólicos en el embrión y el feto. En un estudio 
se observó menor riesgo de obesidad y marcadores de riesgo de enfermedad cardiovascular como insulinemia en ayunas, HOMA y marcadores inflamatorios luego de cirugías malabsortivas y de BGYR por efectos en la descendencia a nivel epigenético y transcripcional ${ }^{35,36,37}$.

Durante el embarazo de una mujer posterior a una cirugía bariátrica pueden presentarse ciertas complicaciones relacionadas con la cirugía: hernias internas, obstrucción intestinal, hiperemesis y colelitiasis. En las pacientes con BGA pueden presentarse problemas con la posición y función de la banda que requieran su revisión. Ante un cuadro de dolor abdominal debe mantenerse un alto nivel de alerta y consultar precozmente con el cirujano bariátrico ${ }^{20}$.

\section{Diagnóstico de diabetes gestacional post-cirugía bariátrica}

La cirugía bariátrica mejora el metabolismo lipídico e hidrocarbonado. La mejoría en el control de la glucemia responde a:

- Post-quirúrgica inmediata: principalmente por el aumento de la sensibilidad hepática a la insulina debido a la restricción de energía postoperatoria y a una mejoría de la función de las células beta.

- En forma mediata: la mejoría en la sensibilidad periférica a la insulina es inducida principalmente por la pérdida de peso ${ }^{32}$.

- El punto a definir es cómo y cuál es la mejor herramienta para el diagnóstico de DG post cirugía bariátrica dado que la prueba de tolerancia oral a la glucosa (PTOG) no puede ser implementada en este grupo de pacientes.

Si bien hay trabajos publicados que mencionan la menor prevalencia de diabetes gestacional (DG) en pacientes post $\mathrm{CB}$ el método de diagnóstico utilizado, PTOG, no sería el adecuado ${ }^{39,40,47}$. Las pacientes con CB presentan una modificación del tracto gastrointestinal que altera la velocidad de absorción de la glucosa ${ }^{45}$, motivo por el cual las poblaciones operadas con CB y no operadas no serían comparables.

Otra dificultad que presenta la realización de la PTOG en el grupo de pacientes post CB es el síndrome de dumping (dolor abdominal, náusea, diarrea, seguidas de hiperinsulinemia, hipoglucemia y síncope) resultado de la liberación de péptidos intestinales como el GLP1, con una alta incidencia de hasta el $60 \%{ }^{41,42,47}$.
Recomendamos no realizar curva de tolerancia oral a la glucosa en pacientes embarazadas luego de una cirugía bariátrica (C).

Si la glucemia plasmática en ayunas es $>0$ igual a $100 \mathrm{mg} / \mathrm{dl}$ en dos determinaciones, se diagnostica DG en cualquier momento del embarazo (C).

La ADA recomienda realizar monitoreo de la glucemia capilar en ayunas y 2 hs postprandial, durante una semana, entre las semanas 24 y 28 de la gestación $n^{32,43,44,47,48,49}$

Nuestra recomendación es realizar monitoreo glucémico 2 hs postprandial durante una semana cuando la glucemia plasmática en ayunas fue < a $100 \mathrm{mg} / \mathrm{dl}$. Si la paciente presenta los siguientes factores de riesgo para DG recomendamos realizar monitoreo glucémico antes de la semana $24^{50}$.

\section{Factores de riesgo}

1. Obesidad.

2. Antecedente de diabetes gestacional.

3. Hijos macrosómicos.

4. Antecedentes familiares de diabetes tipo 2.

Si el $20 \%$ o más de los valores del monitoreo postprandial a las $2 \mathrm{hs}$ desde el inicio de la comida es mayor o igual a $120 \mathrm{mg} / \mathrm{dl}$ se considera DG y se comienza seguimiento y tratamiento siguiendo las guías que se utilizan en pacientes no operadas ${ }^{46,50}$.

Para diagnóstico recomendamos no utilizar la medición de $\mathrm{Hb}$ glicosilada considerando que no hay valores de corte para su uso en el embarazo ${ }^{47,50}$.

Diagnóstico de diabetes gestacional (C):

- Dos glucemias plasmáticas en ayunas $>100 \mathrm{mg} / \mathrm{dl}$ en cualquier momento del embarazo.

- Monitoreo capilar 2 hs pp $>120$ mg/dl durante una semana entre la semana 24-28.

- Si tiene factores de riesgo para diabetes gestacional realizar monitoreo capilar antes de la semana 24 , si es normal entre la semana 24- 28 y repetir en la semana 31-33 (C). 


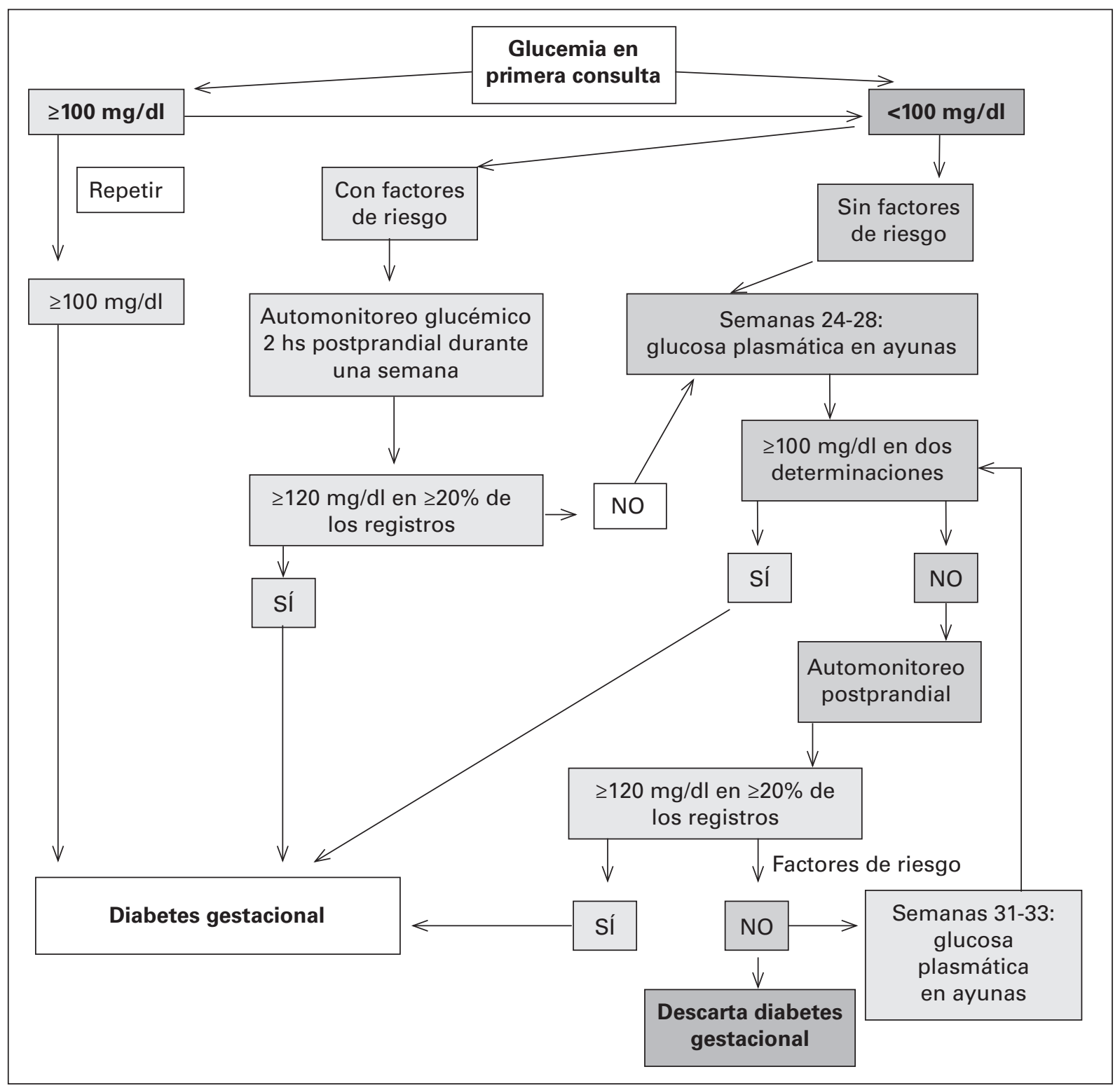

Gráfico 1: Algoritmo diagnóstico de diabetes gestacional para mujeres con cirugía bariátrica previa.

\section{Anticoncepción en mujeres sometidas a cirugía bariátrica}

La fertilidad se encuentra afectada negativamente en la obesidad, por ello las mujeres que han perdido peso luego de una cirugía bariátrica experimentan una mejoría de la fertilidad y pueden estar en riesgo de un embarazo no planeado.

La elección de un método anticonceptivo en pacientes con cirugía bariátrica requiere de una consideración cuidadosa para garantizar que sea eficaz y aceptable para la mujer y evite el embarazo ${ }^{51}$.

Se debe proporcionar un asesoramiento adecuado respecto de la anticoncepción y el embarazo a la paciente que se someterá a una cirugía, independientemente de si desea o no un futuro embarazo. El momento óptimo para este asesoramiento es previo al procedimiento quirúrgico ${ }^{51,52}$.

\section{Anticonceptivos luego de la cirugía bariátrica}

La absorción de los anticonceptivos puede encontrarse afectada luego de la cirugía bariátrica; esta alteración es consecuencia de trastornos gastrointestinales, vómitos y diarrea, en general son consecuencia de una mala elección de alimentos. Otras causas podrían ser alimentos atrapados (bandas y mangas), estenosis de la anastomosis, estrechamiento de la nueva conexión entre 
el estómago e intestino delgado (BGYR).

La farmacocinética de los anticonceptivos orales se ve afectada también por el tipo de procedimiento realizado. Los estrógenos orales se absorben principalmente en el tracto gastrointestinal superior y en la cirugía bariátrica, particularmente en el BGYR se altera la fisiología digestiva ${ }^{51}$.

Los estudios sugieren que la anticoncepción oral puede no ser eficaz como resultado de la disminución de la absorción ${ }^{51,55,56}$.

Desafortunadamente la evidencia actual es limitada debido a la falta de pruebas de eficacia y seguridad.

El American College of Obstetricians and Gynecologists (ACOG) no recomienda el uso de los anticonceptivos orales (ACO) como único método anticonceptivo, vinculando esta recomendación a la malabsorción generada por la resección del intestino delgado, las diarreas y los vómitos ${ }^{12}$.

Con respecto a la farmacocinética, los niveles plasmáticos de hormonas son menores después de la ingestión de norestisterona y levonorgetrel en las pacientes sometidas a bypass yeyunoileal, al compararlo con el grupo control. La explicación más probable para los niveles más bajos hormonales luego del procedimiento quirúrgico es que la absorción se encuentra alterada por la operación. Este hallazgo hace que sea más probable que el riesgo de fracaso al anticonceptivo oral sea mayor en las pacientes operadas con cirugía malabsortiva ${ }^{51,55,56}$.

Los anticonceptivos progestágenos solos y la anticoncepción de emergencia en pacientes con procedimientos malabsortivos puede disminuir su absorción por lo cual no se recomiendan ${ }^{51,57}$.

No se encontró evidencia de disminución de eficacia de la anticoncepción oral en procedimientos restrictivos ${ }^{57}$.

\section{Uso de anticoncepción no oral luego de una cirugía bariátrica}

- Parches transdérmicos

Las pacientes experimentan pérdida de peso significativa luego de una cirugía, esto lleva a que la piel se afloje y pueda generar dificultad para encontrar un lugar adecuado para su adherencia.

- Implantes subdérmicos

Podrían plantear dificultad en su implantación por los cambios en la tonicidad de la piel. Se ha demostrado que los niveles séricos de etonogestrel disminuían con la pérdida de peso, pero dichas concentraciones hormonales están por encima de la concentración mínima requerida para tener un efecto anticonceptivo eficaz ${ }^{51,54}$.

- Anillos vaginales, condones de mujeres, diafragma y capuchones vaginales

Son difíciles de colocar en pacientes obesas y también de controlar su adecuada ubicación. Además el diafragma debe ser controlado para evaluar si es correcto el diámetro y la colocación luego de una pérdida de $3 \mathrm{Kg}$, haciendo esta opción inviable para las pacientes post-cirugía.

\section{- Anticonceptivos inyectables}

Se cree que la asociación de ganancia de peso con los métodos inyectables puede no ser aceptada para las pacientes con cirugía bariátrica.

Además existe una potencial pérdida de densidad ósea con los ACO inyectables que también podría ocurrir con procedimientos bariátricos. Esto se encuentra aún bajo investigación, por lo cual este método debe ser considerado con cautela en la actualidad. Los métodos de esterilización se deben evaluar cuidadosamente según el caso ${ }^{51}$.

- Métodos anticonceptivos sugeridos para pacientes con cirugía bariátrica

Los dispositivos intrauterinos (DIU hormonales o de cobre) se deberían ofrecer como método anticonceptivo de primera línea para las pacientes con cirugía bariátrica.

La anticoncepción ofrecida por el DIU es reversible y no se ve afectada por la pérdida de peso impuesta por la intervención quirúrgica. Si bien los hilos del DIU pueden ser difíciles de comprobar hasta que se logre una pérdida de peso significativa. Los preservativos masculinos son otra opción valedera ${ }^{51,53}$.

\section{CONCLUSIONES}

El aumento de la incidencia de obesidad implica un incremento en el número de cirugías bariátricas realizadas y cada vez a edades más tempranas.

Debemos tener en cuenta que de acuerdo al tipo de cirugía realizada hay que dar pautas concretas para el control de la mujer desde el período preconcepcional, durante el embarazo y posteriormente al mismo.

Un equipo multidisciplinario es obligatorio para cumplir el objetivo de la mejor atención posible asegurando que los resultados de la gestación sean iguales a los de la población general, si bien se reconoce que la tasa de cesárea varía en esta población. La elección del método anticonceptivo debe ser cuidadosa para garantizar su eficacia. 


\section{REFERENCIAS}

1. James WP. WHO recognition of the global obesity epidemic. International Journal of Obesity (2005) 2008, 32 Suppl 7, S120-126.

2. Sirimi N, Goulis DG. Obesity in pregnancy. Hormones 2010, 9, 299-306.

3. Mitchell S, Shaw D. The worldwide epidemic of female obesity. Best practice \& research. Clinical Obstetrics \& Gynaecology 2015, 29, 289-299.

4. Racusin D, Stevens B, Campbell G, Aagaard KM. Obesity and the risk and detection of fetal malformations. Seminars in perinatology 2012, 36, 213-221.

5. Encuesta Nacional de Factores de Riesgo, ENFR 2013. Ministerio de Salud de la Nación.

6. Weiss JL, Malone FD, Emig D, Ball RH, et al. Obesity, obstetric complications and cesarean delivery rate. A population-based screening study. Am. J. Obstet. Gynecol. 2004, 190, 1091-1097.

7. Morgan ES, Wilson E, Watkins T, Gao F, Hunt BJ. Maternal obesity and venous thromboembolism. International Journal of Obstetric Anesthesia 2012, 21, 253-263.

8. Marik PE, Plante LA. Venous thromboembolic disease and pregnancy. N. Engl. J. Med. 2008, 359, 2025-2033.

9. Catalano PM. Management of obesity in pregnancy. Obstet. Gynecol. 2007, 109, 419-433.

10. Cedergren MI. Maternal morbid obesity and the risk of adverse pregnancy outcome. Obstet. Gynecol. 2004, 103, 219-224.

11. Álvarez MGR, y col. Revista de la Sociedad Argentina de Diabetes 2013; 47:69-80.

12. American College of Obstetritian and Gynecologist, ACOG. Practice Bulletin $\mathrm{N}^{\circ}$ 105: Bariatric surgery and pregnancy. Obstet. Gynecol. 2009,113:1405-1413.

13. Magdaleno RJr, Pereira BG, Chaim EA, Turato ER. Pregnancy after bariatric surgery: a current view of maternal, obstetrical and perinatal challenges. Arch. Gynecol. Obstet. 2012 Mar; 285(3):559-66.

14. Beard J, et al. Reproductive considerations and pregnancy after bariatric surgery: current evidence and recommendations. Obes. Surg. 2008: 18 1023-1027.

15. Consenso Argentino de Nutrición en Cirugía Bariátrica. Actualización en Nutrición. 2011, Junio. Vol. 12. № 2.

16. Kominiarek MA. Semin. Perinatol. 2011; 35(6): 356-361.

17. Patel JA, Patel NA, Thomas RL, Nelms JK, Colella JJ. Pregnancy outcomes after laparoscopic Roux-en-Y gastric bypass. Surg. Obes. Relat. Dis. 2008 Jan/feb;4(1):39-45.

18. Printin K J, Scott D. Pregnancy following gastric bypass for the treatment of morbid obesity. American Journal of Surgery, 1982, 4 8: 363-365.

19. Weiss HG, Nehoda H, Labeck B, Hourmont K, Marth C, Aigner F. Pregnancies after adjustable gastric banding. Obesity Surgery, 2001, 11:303-306.

20. Willis $K$, Lieberman N, Sheiner E, et al. Pregnancy and neonatal outcome after bariatric surgery. Best Practice \& Research Clinical Obstetrics and Gynaecology 2015 (29): 133-144.

21. Sheiner $E$, Edri A, Balaban E, et al. Pregnancy outcome of patients who conceive during or after the first year following bariatric surgery. Am. J. Obstet. Gynecol. 2011; 240:50e1-6.

22. Wax JR, Cartin A, Wolff R, Lepich S, Pinette MG, Blackstone J. Pregnancy following gastric bypass for morbid obesity: effect of surgery-to-conception interval on maternal and neonatal outcomes. Obes. Surg. 2008; 18(12):1517.
23. Haward RN, Brown WA. Does pregnancy increase the need for revisional surgery afterlaparoscopic adjustable gastric banding? Obes. Surg. Aug 1.2010 published online.

24. Lapolla A, Marangon M, Dalfra MG, et al. Pregnancy outcome in morbidly obese women before and after laparoscopic gastric banding. Obes. Surg. 2010; 20:1251-1257.

25. Johansson K, Cnattingius S, Näslund I, et al. Outcomes of pregnancy after bariatric surgery. N. Engl. J. Med. 2015; 372: (9) 814-824.

26. Wax JR, Cartin A, Wolff R, et al. Pregnancy following gastric bypass surgery for morbid obesity: effect of surgery to conception interval on maternal and neonatal outcomes. Obes. Surg. 2008:18:540-544.

27. Lesk J, Peaceman A. Pregnancy outcomes in women after bariatric surgery compared with obese and morbidly obese controls. Obstetrics \& Gynecology. March 2012, Vol. 119, Issue 3, p 547-554.

28. Bebber FE, Rizzolli J, Casagrande DS, et al. Pregnancy after bariatric surgery: 39 pregnancies follow-up in a multidisciplinary team. Obes. Surg. 2010 Sep 05.

29. Woodard CB. Pregnancy following bariatric surgery. Journal of Perinatal and Neonatal Nursing, Vol. 18, N ${ }^{\circ}$ 4, pp. 329-340, 2004.

30. Stephansson K, et al. Outcomes of pregnancy after bariatric suregry. N. Engl. J. Med. 372:9 pp. 814-824, 2015.

31. Kaska L, Kobiela J, Abacjew-Chmylko A, et al. Nutrition and pregnancy after bariatric surgery. ISRN Obesity. Vol 2013, article ID 492060, 6 pages. Review http://www.hindawi.com/journals/ isrn/2013/492060/ ultima vez visto 21/6/2015.

32. Uzoma A, Keriakos R. Pregnancy management following bariatric surgery. Journal of Obstetrics and Gynaecology, February 2013; 33: 109-114. Review.

33. Decker GA, Swain JM, Crowell MD, Scolapio JS. Gastrointestinal and nutritional complications after bariatric surgery. American Journal of Gastroenterology, Vol. 102, № 11, pp. 2571-2580, 2007.

34. Nomura RM, Dias MC, Igai AM, Paiva LV, Zugaib M. Anemia during pregnancy after silastic ring Roux-en-Y gastric bypass: influence of time to conception. Obes. Surg. 2011;21:479-484.

35. Smith J, Cianflone K, Biron S, et al. Effects of maternal surgical weight loss in mothers on intergenerationl transmission of obesity. J. Clin. Endocrinol. Metab. 2009;94:4275-83.

36. Guenard F, Patty ME. Reducing maternal weight improves offspring metabolism and alters(or modulates) methylation. PNAS 2013, 110: 32, 12859-12860.

37. Kral JG, et al. Large maternal weight loss from obesity surgery prevents transmission of obesity to children who were followed for 2 to 18 years. Pediatrics 118:e1644-e1649. 2006.

38. Gadgil $\mathrm{M}$, et al. Laboratory testing for and diagnosis of nutritional deficiencies in pregnancy regnancy before and after bariatric surgery. J. Womens Health (Larchmt). 2014 Feb 1; 23(2): 129-137.

39. Burke $A E$, Bennett WL, Jamshidi RM, et al. Reduced incidence of gestational diabetes with bariatric surgery. J. Am. Coll. Surg. 2010;211:169-75.

40. Kominiarek MA. Preparing for and managing a pregnancy after bariatric surgery. Semin Perinatol. 2011 December ; 35(6): 356361

41. Scheiner E, et al. Pregnancy outcome of patients with gestational diabetes mellitus following bariatric surgery. American Journal of Obstetrics and Gynecology (2006) 194, 431-5. 
42. Buchwald H, AvidorY, Braunwald E, Jensen MD, Pories W, Fahrbach $\mathrm{K}$, et al. Bariatric surgery: a systematic review and metaanalysis. JAMA 2004; 292:1724-37.

43. Wax JR, Pinette MG, Cartin A, Blackstone J. Female reproductive issues following bariatric surger. Obstet. Gynecol. Surv. 2007; 62:595.

44. American Diabetes Association. Gestational diabetes mellitus. Practice Guideline. Diabetes Care 2004; 27: 88-90.

45. Roslin MS, Oren JH, Polan BN, Damani T, Brauner R, Shah PC. Abnormal glucose tolerance testing after gastric bypass. Surg. Obes. Relat. Dis 2013; 9:26.

46. Recomendaciones para la gestante con diabetes. Revista de la Sociedad Argentina de Diabetes. Vol. 43, N²:73-81, 2009.

47. Freitas $\mathrm{C}$. Effect of new criteria on the diagnosis of gestational diabetes in women submitted to gastric bypass surgery for obesity and related diseases 2014, 10 (6): 1041-1046.

48. Guelinckx I. Reproductive outcome after bariatric surgery: a critical review (Review). Human Reproduction Update, Vol. 15, Issue 2, 2009, pages 189-201.

49. Apovian CM, Cummings S, Anderson W, et al. Best practice updates for multidisciplinary care in weight loss surgery. Obesity (Silver Spring) 2009; 17:871.

Nota: en relación a los aspectos metodológicos (búsqueda, selección y evaluación bibliográfica, establecimiento de niveles y grados de evidencia, formulación de las recomendaciones, etc.) se remite a los lectores al "Manual Metodológico para la Elaboración de Guías de Práctica Clínica
50. NICE Guideline. Published: 25 February 2015nice.org.uk/guidance/ng3.

51. Grahan Y, Wilkes S, Mansour D, Small PK. Contraceptivo needs of women following bariatric surgery. Fam. Plann Reprod. Health Care 2014 40:241-244.

52. Paulen ME, Zapata LB, Cansino C, et al. Contraceptive use among women with a history of bariatric surgery: a systematic review. Contraception 2010; 82:86.

53. Hilman JB, Miller RJ, IngeTH. Menstrual concerns and intrauterine contraception among adolescent bariatric surgery patients. Journal of Women's Health, Vol. 20, N 4, 2011.

54. Ciangura $C$, Corigliano N, Basdevant A, et al. Etonogestrel concentration in morbidly obese woman following Roux-en- $Y$ gastric bypass surgery: three case reports.

55. Zaher O, Merhi MD. Challenging oral contraception after weight loss by bariatric surgery. Gynecol. Obstret. Invest. 2007; 64:100102.

56. Zaher O, Merhi MD. Revisiting optimal hormonal contraception following bariatric surgery.Contraception 87 (2013) 131-133.

57. Centers for Disease Control and Prevention. Morbidity and Mortality Weekly Report (MMWR). U.S. Medical Eligibility Criteria for Contraceptive Use, 2010. http://www.cdc.gov/mmwr/preview/ mmwrhtml/rr59e0528a1.htm (Accessed on May 28, 2010).

en el Sistema Nacional de Salud" del Programa de Guías de Práctica Clínica del Sistema Nacional de Salud de España http://portal.guiasalud.es/ emanuales/elaboracion/documentos/Manual\%20 metodologico \% 20-\%20Elaboracion \%20GPC \% 20 en $\% 20$ el\%20SNS.pdf. 